\title{
Überempfindlichkeit auf Jod: Rien ne va plus?
}

\section{A. J. Bircher}

\section{Hypersensitivity to lodine - Rien ne va plus?}

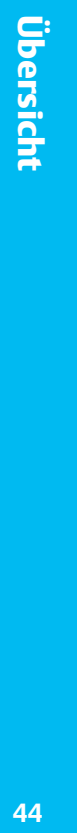

\section{Zusammenfassung}

Eine Jodallergie wird im Alltag häufig vermutet, ist aber relativ selten. Insbesondere auf Jodderivate zurück zu führende Überempfindlichkeitsreaktionen sind heutzutage rar geworden. Das anorganische Jodid $\left(\mathrm{I}^{-}\right)$ist weniger reaktiv, das molekulare Jod $\left(\mathrm{I}_{2}\right)$ ist stark reaktiv, wärend das Jod in Kontrastmitteln inert ist, solange es kovalent gebunden bleibt. Öfters werden pseudoallergische Reaktionen auf jodhaltige Röntgenkontrastmittel beobachtet, die aber nicht durch das Jod ausgelöst werden, sondern von der Osmolalität des Kontrastmittels abhängen. Als pathophysiologischer Mechanismus werden hier Komplementaktivierung und eine direkte Aktivierung von basophilen Granulozyten und Mastzellen verantwortlich gemacht. Gelegentlich werden kontaktallergische Reaktionen auf das Povidon-Jod beobachtet, die teilweise einen vesikulo-bullösen Aspekt annehmen können. Aber auch toxisch-irritative kutane Reaktionen auf Jod können massiv bullös verlaufen. Einen immunologischen Pathomechanismus scheinen die allergischen Arzneimittel-Exantheme auf Röntgenkontrastmittel zu haben, die als T-Zell-vermittelte Reaktionen verstanden werden. Die auslösenden Antigene sind nicht eindeutig erklärt, gemäß neueren Daten handelt es sich wahrscheinlich um die Röntgenkontrastmoleküle selbst, und nicht um das in Spuren vorhandene Jodid. IgE-mediierte anaphylaktische Reaktionen auf Povidon-Jod wurden selten beschrieben, hier scheint Povidon selbst das auslösende Antigen zu sein. Einige so genannte idiosynkratische Reaktionen, bei welchen der Pathomechanismus unklar ist, werden heute nur noch seltenerweise beobachtet. Dazu werden die Jod-Akne, das akneiforme JodExanthem, das tuberöse Jododerm sowie die Jod-Sialoadenitis gezählt, die wahrscheinlich durch Jodide ausgelöst werden. Noch seltener sind Überempfindlichkeitsreaktionen wie schwere
Abstract

Suspicion of allergy to iodine is a commonly suspected clinical problem, however, often it is not sufficiently documented. Hypersensitivity reactions to iodine derivatives are currently rare. More common are pseudoallergic reactions to iodinated contrast media, however these reactions are dependent on the osmolality of the contrast media and not on the iodine content.

Delayed allergic reactions to iodinated contrast media include exanthemas where a T-cell mediated pathophysiology has been proposed. The eliciting antigens are not known, recent data indicate that probably the molecules itself and not iodine are involved. Occasionally, toxic bullous reactions and contact allergy to povidone iodine have been observed. IgE-mediated anaphylactic reactions from povidone iodine have been attributed to the povidone molecule and not to the iodine itself. Idiosyncratic reactions where pathophysiological mechanisms are mostly unknown are only rarely observed. These include iodine-induced acne, acneiforme exanthemas, iododerma and iodine mumps. Very rarely, febrile exanthemas and severe bullous exanthemas have been reported from iodine intake. Patients with dermatitis herpetiformis Duhring and hypocomplementemic vasculitis experienced an exacerbation of the disease upon exposure to high iodine doses. Hypersensitivity reactions to iodide in food have only rarely been reported to seaweed-containing food. Because of the very different pathomechanisms involved in the different hypersensitivity reactions to iodine and iodide containing drugs and diagnostic media, a general ,iodine allergy” does not exist. Based on a standardized and complete allergologic investigation safe alternatives may be identified in such patients.

Institutsangaben

Allergologische Poliklinik, Dermatologische Klinik, Universitätsspital Basel/Schweiz

Korrespondenzadresse

Prof. Dr. med. Andreas J. Bircher · Allergologische Poliklinik · Dermatologische Klinik · Universitätsspital Basel · Petersgraben 4·4031 Basel · Schweiz·E-mail: andreas.bircher@unibas.ch

Bibliografie

Akt Dermatol 2006; 32: 44-49 @ Georg Thieme Verlag KG Stuttgart • New York

DOI 10.1055/s-2005-921136 · ISSN 0340-2541 
febrile Exantheme und schwere bullöse Dermatosen geworden. Patienten mit einer Dermatitis herpetiformis Duhring sowie mit einer hypokomplementämischen Vaskulitis erleiden eine Exazerbation ihrer Grundkrankheit auf höhere Joddosen. Hingegen sind auf jodhaltige Nahrungsmittel wie Meerfische und Meeresfrüchte trotz ihrem Jodgehalt keine allergischen Reaktionen zu erwarten. Aufgrund der sehr unterschiedlichen Pathomechanismen der Überempfindlichkeitsreaktionen auf Jod und Jodide enthaltende Arzneimittel und Diagnostika, liegt nie eine allgemeine „Jodallergie“ vor. Mit einer umfassenden allergologischen Abklärung können diese aufgeklärt und in deren Folge andere jodhaltige Arzneimittel wieder problemlos eingesetzt werden.

\section{Einleitung}

Individuen mit einer angeblichen generellen „Jodallergie“ sind in der täglichen Praxis nicht selten, oft haben solche Patienten eine unerwünschte Wirkung nach Kontrastmitteluntersuchungen erlitten. Eine unkritische verallgemeinernde Etikettierung kann aber weitreichende Konsequenzen haben, insbesondere der Einsatz von jodhaltigen Kontrastmitteln wird erschwert. Auch in einer Umfrage unter Ärzten eines Universitätsspitals war die Kenntnis über unerwünschte Wirkungen, Risiken für Kontrastmittelreaktionen und die Unbedenklichkeit von jodhaltigen Medikamenten, Desinfektions- und Nahrungsmittel bei solchen Patienten nur sehr beschränkt vorhanden [1].

Wie oft bei Überempfindlichkeitsreaktionen, werden unter dem Begriff der „Jodallergie“ die unterschiedlichsten unerwünschten Reaktionen subsumiert. Wie bei allen allergischen Krankheiten ist aber dem Auslöser, dem Typ der allergischen Reaktion, der genauen Symptomatik und allenfalls vorgenommenen Diagnostik große Beachtung zu schenken, um die Patienten nicht unnötigerweise von wichtigen diagnostischen Prozeduren auszuschließen oder ihnen Therapeutika vorzuenthalten. Im vorliegenden Artikel werden die Überempfindlichkeitsreaktionen auf jodhaltige Medikamente dargestellt und auf die Frage der Auslösung von unerwünschten Wirkungen durch Jod eingegangen.

Jod (vom griechischen iodes = violett), ein Halogen mit einem Atomgewicht von 126.90, ist ein nichtmetallisches Element der Gruppe VII mit der Ordnungzahl 53. Chemisch aktiv ist vor allem das elementare $\mathrm{J}_{2}$, wie es in Desinfektionsmitteln eingesetzt wird. Die ionisierte Form des Jod $\left(\mathrm{J}^{-}\right)$, das als Jodid in verschiedenen Verbindungen vorkommt, ist wenig reaktiv. Jod ist ein essenzielles Spurenelement, die täglich empfohlene Dosis beträgt etwa $90 \mu \mathrm{g}$ für ein Kind und bis zu $150 \mu \mathrm{g}$ für einen Erwachsenen. In der Schwangerschaft und der Stillzeit werden tägliche Dosen von 220-290 $\mu \mathrm{g}$ empfohlen. Die maximale täglich empfohlene Dosis liegt bei $200 \mu$ g für Kinder und $1100 \mu$ g für Erwachsene.

Jod wird über die Nahrung aufgenommen, so enthält z.B. ein Hühnerei ca. $25 \mu \mathrm{g}$, ein Glas Milch ca. $50 \mu \mathrm{g}$ Jod. Besonders jodreich sind vor allem Meersalz, Meerfische (ca. $100 \mu \mathrm{g} / 100 \mathrm{~g}$ ) sowie getrockneter Kelp, der bis zu $170 \mathrm{mg} / 100 \mathrm{~g}$ [2] enthalten kann. Früher waren aufgrund eines Jodmangels die Struma, die Hypothyreose sowie der Kretinismus in weiten Gebieten Europas endemisch.

\section{Medizinische Anwendungen}

Jod wurde und wird in der Medizin häufig als Diagnostikum und Therapeutikum eingesetzt. Schon um 1880 wurde vom Franzosen Casimir Davaine die bakterizide Wirkung des Jods entdeckt. Am Anfang des 20. Jahrhunderts waren deshalb jodhaltige Desinfektionsmittel weit verbreitet. Die früher oft verwendete Jodtinktur enthält 9 Teile Jod auf Ethanol und Wasser, insgesamt $65 \mathrm{mg}$ Jod und $19 \mathrm{mg}$ Jodid pro Gramm. Die 5\% Lugol'sche Lösung enthält $56 \mathrm{mg}$ Jod und $85,7 \mathrm{mg}$ Jodid (als Kaliumjodid) pro ml und wurde früher z. B. in der systemischen Behandlung der Lues und des Erythema nodosum eingesetzt.

Ab ca. 1950 wurde das mit Polyvinylpyrrolidon komplexierte elementare Jod eingeführt. Daraus wird das bakterizide Jod kontinuierlich abgegeben. Damit konnte sowohl die Freisetzung, die Wirkung als auch die Gewebepenetration optimiert werden. Konzentrationen von Povidon-Jod liegen zwischen 5-10\% $(5-10 \mathrm{mg} / \mathrm{ml})$. Das Jod und Chlor enthaltende Desinfiziens Chlorjodhydroxychinolin (Vioform ${ }^{\circledR}$ ), wird heute praktisch nicht mehr verwendet.

Neben der Wund- und Hautdesinfektion wird heute Jod vor allem in der Diagnostik in Form der jodhaltigen Kontrastmittel eingesetzt, in der Nuklearmedizin als radioaktives Jod-123 und Jod-131 diagnostisch, als Jod-131 auch therapeutisch verabreicht, sowie als topische Formulierung bei der so genannten Minor'schen Schweißprobe und mit der Schiller'schen Jodprobe bei der Identifikation von Zervixdysplasien verwendet. Kontrastmittel enthalten kovalent gebundenes elementares Jod, deshalb liegt Jodid als Kontamination nur in bis zu $25 \mu \mathrm{g} / \mathrm{ml}$ vor (persönliche Mitteilung Catherine Christiansen, Amersham Health, Norwegen).

Therapeutika die Jod enthalten, sind z.B. einige Expektorantien und Mukolytika, die bis zu $10 \mathrm{mg} / \mathrm{ml}$ enthalten können, Thyroxin das zur Substitution bei der Hypothyreose eingesetzt wird, sowie Amiodaron, ein Antiarrhythmikum. Kaliumjodid wird als Notfallmedikament zur Blockade der Schilddrüse bei Strahlungsunfällen gelagert. Viele Vitamin- und Aufbaupräparate enthalten zudem Jod. Die zugeführten Joddosen können einige Mikro-

Tab. 1 Jodgehalt in verschiedenen Nahrungsmitteln und Medikamenten (nach [3])

\begin{tabular}{|c|c|}
\hline jodiertes Kochsalz & $15 \mathrm{mg} / \mathrm{kg}$ \\
\hline Erythrosin E 127 & $\begin{array}{l}577 \mathrm{mg} / \mathrm{g} \text { (mittlere Tagesdosis } 10 \mu \mathrm{g} \\
\text { Jod) [44] }\end{array}$ \\
\hline jodiertes Röntgenkontrastmittel & $\begin{array}{l}150 \text { bis } 370 \mathrm{mg} / \mathrm{ml} \text { elementares Jod } \\
\text { Jodid bis ca. } 25-50 \mu \mathrm{g} / \mathrm{ml} \\
\text { (bei Licht- und Strahlenexposition } \\
\text { evtl. höher) }\end{array}$ \\
\hline $\begin{array}{l}\text { Tabletten, Tropfen } \\
\text { Lugol'sche Lösung 5\% } \\
\text { Amiodaron } \\
\text { Expectorans (Perpector }{ }^{\circledR} \text { ) } \\
\text { Kaliumjodidtabletten } \\
\text { Kelp-Tabletten }\end{array}$ & $\begin{array}{l}\text { Jod } 56 \mathrm{mg} / \mathrm{ml} \text {, Jodid } 85 \mathrm{mg} / \mathrm{ml} \\
\text { Jod } 75 \mathrm{mg} \text { pro Tablette à } 200 \mathrm{mg} \\
10 \mathrm{mg} / \mathrm{ml} \\
65 \mathrm{mg} \text { pro Tablette } \\
0,05 \text { bis } 0,15 \mathrm{mg} \text { pro Tablette }\end{array}$ \\
\hline $\begin{array}{l}\text { topische Antiseptika } \\
\text { Povidon-Jod (Betadine }{ }^{\circledR} \text { ) } \\
\text { Jodtinktur }\end{array}$ & $\begin{array}{l}\text { PVP-Jod } 10 \mathrm{mg} / \mathrm{ml} \\
\text { Jod } 65 \mathrm{mg} / \mathrm{ml} \text {, Jodid } 19 \mathrm{mg} / \mathrm{ml}\end{array}$ \\
\hline
\end{tabular}


Tab. 2 Überempfindlichkeitsreaktionen auf jodhaltige Präparate/Nahrungsmittel

\begin{tabular}{|c|c|c|c|c|c|}
\hline Typ/Mechanismus & Klinik & Auslöser & Auslösendes Allergen & Diagnostik & Kontraindikation für Jod \\
\hline Typ IV & allergisches Kontaktekzem & $\begin{array}{l}\text { Jodtinktur } \\
\text { Povidon-Jod }\end{array}$ & Jod & $\begin{array}{l}\text { Epikutantest } \\
\text { Epikutantest }\end{array}$ & $\begin{array}{l}\text { topisches Jod } \\
\text { Povidon-Jod }\end{array}$ \\
\hline Typ IV & Exanthem makulopapulös & Kontrastmittel & $\begin{array}{l}\text { Kontrastmittelmolekül } \\
\text { ev. Jod }\end{array}$ & $\begin{array}{l}\text { Epikutantest } \\
\text { Intradermaltest } \\
\text { (ev. Provokation) }\end{array}$ & auslösende Kontrastmittel \\
\hline Typ I & Urtikaria, Anaphylaxie & Povidon-Jod & Povidonmolekül & Prick, Intradermaltest & povidonhaltige Topika \\
\hline Pseudoallergie & anaphylaktoide Reaktion & $\begin{array}{l}\text { Kontrastmittel- } \\
\text { molekül }\end{array}$ & $\begin{array}{l}\text { Kontrastmittel } \\
\text { ionische }>\text { nichtionische }\end{array}$ & $\begin{array}{l}\varnothing \\
\text { (ev. Provokation) }\end{array}$ & $\begin{array}{l}\text { auslösende Kontrastmittel } \\
\text { (v.a. ionische) } \\
\text { ev. Prämedikation }\end{array}$ \\
\hline Idiosynkrasie & $\begin{array}{l}\text { Jododerm } \\
\text { Jodsialoadenitis } \\
\text { Thyroiditis (Amiodaron) } \\
\text { Dermatitis herpetiformis Duhring } \\
\text { hypokomplementämische Vaskulitis } \\
\text { neutrophile Vaskulitis }\end{array}$ & $\begin{array}{l}\text { Kaliumjodid } \\
\text { Amiodaron }\end{array}$ & Jodid & (ev. Provokation) & $\begin{array}{l}\text { topisches und systemisches } \\
\text { Jod (hochdosiert) }\end{array}$ \\
\hline Typ I & Nahrungsmittelallergie & $\begin{array}{l}\text { Fische } \\
\text { Meeresfrüchte } \\
\text { Meersalz }\end{array}$ & Nahrungsproteine & Prick, lgE & keine \\
\hline Pseudoallergie & Scombroidintoxikation & $\begin{array}{l}\text { Scombroideae } \\
\text { (z.B. Thunfisch) }\end{array}$ & $\begin{array}{l}\text { Scombrotoxin } \\
\text { (= Histamin) }\end{array}$ & $\begin{array}{l}\text { Histaminmessung } \\
\text { im Fisch }\end{array}$ & keine \\
\hline
\end{tabular}

gramm bis zu mehreren Gramm (Tab.1) betragen [3], wobei für eine biologische Wirkung wiederum die chemische Form entscheidend ist.

\section{Toxische Reaktionen}

Jod und seine Derivate können eine ganze Reihe von unerwünschten Wirkungen hervorrufen. Dabei stehen primär akute irritative und korrosive Effekte an der Haut und dem Gastrointestinaltrakt im Vordergrund. Bei oraler Aufnahme tritt in der Mundhöhle ein metallischer, bitterer Geschmack auf. Ösophagitis und Gastritis können bei schweren Verätzungen beobachtet werden. Bei der Inhalation von Joddämpfen können ein Ödem der Glottis und ein Lungenödem auftreten. Höhere Dosen können zum Kreislaufschock führen. Mit einer chronischen Toxizität ist ab $2 \mathrm{mg}$ pro Tag zu rechnen, ab ca. $3 \mathrm{~g}$ Jod per os wurden letale Ausgänge beobachtet. Jod kann auch auf der Haut irritative Dermatitiden und starke Verätzungen hervorrufen [4,5]. Die perkutane Absorption von Jod kann erheblich sein und zu systemischen toxischen oder allergischen Symptomen führen.

Die subakute und chronische Toxizität ist vor allem durch unerwünschte Wirkungen auf die Schilddrüse gekennzeichnet. Hohe Joddosen können sowohl zu Hypo- als auch zu Hyperthyreoidismus führen. In Einzelfällen wurden derartige Komplikationen nach Kontrastuntersuchungen, die Patienten hatten mittlere kumulative Joddosen von $21 \mathrm{~g}(1-61 \mathrm{~g})$ erhalten [3], und nach Amiodaron, das zu einer Jodzufuhr von $6 \mathrm{mg} /$ Tag führen kann [6], beobachtet.

\section{Überempfindlichkeitsreaktionen}

\section{Allergische Reaktionen \\ Allergische Reaktionen vom Typ IV}

Kontaktallergische Reaktionen auf Jod und Jodsalze sind schon lange bekannt. Albert Neisser beschrieb 1884 Jodoform-Exantheme und Bruno Bloch, der frühere Vorsteher der Dermatologischen Abteilung in Basel, hat schon 1911 eine ausführliche experimentelle Arbeit über die Jodoform-Idiosynkrasie und die darauf beruhende akute Dermatitis publiziert [7]. Diese klassischen allergischen Kontaktekzeme zeigten häufig einen vesikulobullösen Verlauf und führten nicht selten zur Dissemination. Jod und seine Derivate gehörten zusammen mit den Quecksilbersalzen zweifellos zu den häufigsten iatrogenen Kontaktallergenen in der ersten Hälfte des 20. Jahrhunderts. Später war das Jod selbst und das chlor- und jodhaltige Vioform ${ }^{\circledR}$ ein häufiges Kontaktallergen, wobei beim letzteren Jod nicht das eigentlich Allergen darstellt. Allergische Kontaktekzeme sind seit der Einführung des mit Polyvinylpyrrolidon komplexierten Jodes selten geworden [8,9]. Die Epikutantestung mit Povidone-Jod und Jodtinktur sollte aber mit geeigneten, nicht-irritativen Testkonzentrationen durchgeführt werden $[10,11]$.

In den letzten Jahren wurde vermehrt über Röntgenkontrastmittel-induzierte Arzneimittelexantheme berichtet (Tab. 2). Diese scheinen eine immunologische T-Zell-vermittelte Pathogenese zu haben [12]. Gemäß neueren Daten ist wahrscheinlich nicht das an sich in den Kontrastmitteln fest gebundene Jod, sondern Kontrastmittelmoleküle das eigentliche Allergen. Eigene Beobachtungen ergaben, dass einige Patienten auf alle jodhaltigen Kontrastmittel und auf die orale Gabe von Lugol'scher Lösung reagierten. Dies würde für eine eigentliche Spättypreaktion auf Jodide sprechen, Kontrastmittel enthalten bis ca $25 \mu \mathrm{g} / \mathrm{ml}$ Jodid. Andere Patienten reagieren hingegen nur auf wenige Produkte 
und tolerieren andere jodhaltigen Kontrastmittel, was die Kontrastmittelmoleküle als eigentliches Allergen vermuten lässt.

Diagnostisch werden Epikutan- oder Intradermalteste mit Spätablesung (24-48 h) eingesetzt [12]. Der prädiktive Aussagewert negativer Teste ist aber relativ gering, d.h. dass bei Reexposition mit test-negativen Röntgenkontrastmitteln Rezidive beobachtet wurden [14]. Als Alternative bietet sich die Verwendung von nicht jodhaltigen Kontrastmitteln wie Gadolinium-Derivaten an $[15,16]$.

Ein Arzneimittelexanthem unter dem Bild eines Baboon-Syndroms oder SDRIFE (symmetrical drug-related intertriginous flexural exanthem) wurden bisher überwiegend auf Antibiotika, insbesondere Amoxicillin, beschrieben [17]. Wir haben einen Patienten beobachtet, der ein derartiges Exanthem auf zwei verschiedene jodhaltige Röntgenkontrastmittel entwickelte. Die Hautteste waren positiv auf die auslösenden Produkte, während eine testnegative Substanz in der Reexposition symptomlos toleriert wurde [18].

\section{Allergische Reaktionen vom Soforttyp}

Echte anaphylaktische Reaktionen, die mit hoher Wahrscheinlichkeit durch IgE-Antikörper vermittelt sind, wurden auf Povidon-Jod nach topischer Applikation beschrieben (Tab. 2) [19]. Es wurden Reaktionen mit Urtikaria bis hin zu schweren anaphylaktischen Schockreaktionen nach Applikation auf der vaginalen Schleimhaut oder der Haut beschrieben [20,21]. Die meisten Patienten wiesen einen positiven Pricktest auf Povidon-Jod, einige auch auf das Povidon selbst auf. Bei einigen Patienten fielen die Hautteste mit Jod selbst negativ aus. Dies weist darauf hin, dass nicht das Jod-Molekül, sondern das Polyvinylpyrrolidon das Antigen darstellt [19]. Diese Annahme beruht vor allem auf dem Vorliegen von positiven Prick- oder Intradermaltesten, seltenerweise wurden auch spezifische IgE gegenüber Povidon nachgewiesen.

\section{Pseudoallergische Reaktionen}

Ungleich häufiger sind, vor allem wegen des weit verbreiteten Einsatzes von jodhaltigen Röntgenkontrastmitteln, anaphylaktoide Reaktionen unter dem Bild von Angioödemen, Urtikaria, Bronchospasmus bis hin zum anaphylaktoiden Schock mit gelegentlich letalem Ausgang. Obwohl einzelne Autoren einen IgEvermittelten Mechanismus vermuten [12,13,19,22], konnte ein solcher bisher nicht schlüssig für die meisten dieser Kontrastmittelreaktionen belegt werden. Als Ursache wird vor allem die Hyperosmolalität der Kontrastmittel vermutet, die Einführung von nicht-ionischen Kontrastmitteln mit einer tieferen Osmolalität hat zu einer Abnahme der Häufigkeit schwerer Reaktionen geführt. Als weitere Mechanismen werden die direkte Aktivierung des Komplementsystems und die nicht-spezifische, d.h. nicht-IgE-vermittelte Histamin-Liberation aus basophilen Granulozyten und Mastzellen vermutet [12,13,19,22].

Bei Patienten die eine anaphylaktoide Reaktion auf ein Röntgenkontrastmittel erlitten haben, besteht ein erhöhtes Risiko, auf andere jodierte Kontrastmittel zu reagieren. Eine Prämedikation mit Antihistaminika und Kortikosteroiden kann allenfalls die Reaktion abschwächen aber nicht sicher verhindern [23]. Die vorgeschlagenen Protokolle sind sehr unterschiedlich und oft an ei- nem heterogenen Patientenkollektiv mit unterschiedlichen Reaktionen überprüft worden [22].

\section{Idiosynkratische Reaktionen}

Da früher Jod als Desinfektionsmittel und in Form des Kaliumjodid bei vielen Krankheiten teils in hohen Dosen eingesetzt wurde, wurden unerwünschte Wirkungen wie die Jodakne, das so genannte Jododerm und der so genannte Jodmumps häufiger beobachtet. Die alte Literatur unterscheidet noch einige anderen morphologischen Manifestationen wie die Jodpurpura, den Jodpemphigus, urtikarielle und schwere bullöse Exantheme [24]. Die Pathogenese dieser Reaktionen ist weitgehend ungeklärt, eine individuelle Disposition scheint eine Rolle zu spielen, deshalb werden diese Reaktionen als Idiosynkrasien klassifiziert.

Bei der Jodakne handelt es sich um ein akneiformes Exanthem, wie es auch auf andere Halogene, wie z.B. Brom und Chlor, beobachtet wird. Geringe über lange Zeiträume eingenommene Joddosen können eine vorbestehende Akne vulgaris verschlechtern. Daneben können de novo follikuläre akneiforme Exantheme, typischerweise ohne Komedonen und an atypischer Lokalisation im Sinne einer Acne toxica oder medicamentosa auftreten [25].

Das Jododerm (Jododerma tuberosum) ist eine neutrophile Dermatose, die sich primär mit Papulopusteln in den seborrhoischen Arealen nach oraler, parenteraler und topischer Verabreichung manifestiert. Vegetierende, infiltrierte Plaques, erosive und nässende Läsionen vor allem im Gesicht und an den Extremitäten können im Verlauf auftreten [26-28]. Einzelne Fälle wurden nach Röntgenkontrastmitteln, oraler Therapie mit Kaliumjodid und einmal nach Amiodaron [27] beschrieben. Vor allem niereninsuffiziente Patienten sind betroffen, selten wurden Lymphome und das multiple Myelom als Risikofaktoren bezeichnet [29], ausnahmsweise wurden Todesfälle beobachtet [26]. Der Pathomechanismus ist unklar, eine hohe Joddosis, vor allem bei Niereninsuffizienz, scheint begünstigend zu sein.

Der so genannte Jodmumps oder die Jodsialoadenitis ist eine ebenfalls sehr seltene unerwünschte Wirkung, bei welcher es ca. einen Tag nach Jodexposition z.B. in Kontrastmitteln zu einer ausgeprägten Schwellung der Speicheldrüsen, insbesondere der Glandula submandibularis und sublingualis, seltener auch der Parotis kommen kann [18,30,31]. Ein gelegentlich vermutetes Angioödem ist differenzialdiagnostisch leicht abzugrenzen.

Histologisch lässt sich lediglich ein ausgeprägtes Ödem mit diskreten, vor allem lymphozytären Zellinfiltraten nachweisen [18]. Die Pathogenese ist hier ebenfalls unklar, es scheint eher ein individuell bedingter, toxischer Mechanismus in Frage zu kommen. Bei einzelnen Patienten liegt eine Niereninsuffizienz vor, bei renalen Eliminationsstörungen können höhere Joddosen über die Speicheldrüsen ausgeschieden werden. Hier scheint Jod bzw. Jodid das auslösende Agens zu sein, wie wir in einem Fall mit oralen Provokationstests mit Lugol-Lösung bestätigen konnten [18].

\section{Andere Überempfindlichkeitsreaktionen}

Ausnahmsweise wurden Patienten mit schweren febrilen Exanthemen, seltenerweise auch Stevens-Johnson-Syndrom, toxi- 
scher epidermaler Nekrolyse [13] und einer kutanen Vaskulitis nach Kontrastmitteln in Kombination mit anderen Überempfindlichkeitsreaktionen $[28,32]$ beschrieben. Patienten mit hypokomplementämischer Vaskulitis [33] und Dermatitis herpetiformis Duhring [34,35] können Schübe ihrer Grundkrankheit nach Gabe von Jod erleiden, deshalb ist bei diesen Erkrankungen die Verwendung von jodhaltigen Medikamenten kontraindiziert.

Amiodaron (Cordarone $^{\circledR}$ ), ein Antiarrhythmikum, kann neben der bekannten Pseudozyanose und phototoxischen Reaktionen, auch schwere Hypersensitivitätspneumopathien auslösen. Ein Fall eines Angioödems auf Amiodaron wurde beschrieben, wobei kein Hinweis besteht, dass hier das Jod eine Rolle spielte [36]. Auf radioaktive Jodverbindungen und Thyroxin wurden bisher keine Überempfindlichkeitsreaktionen im eigentlichen Sinne publiziert.

\section{Jodhaltige Nahrungsmittel}

Gelegentlich wird bei sogenannt Jodüberempfindlichen vermutet, dass sie auch auf jodhaltige Nahrungsmittel, insbesondere Meeresfrüchte und Fische, reagieren. Jod spielt hier praktisch nie eine Rolle. Soforttyp-Reaktionen auf Meeresfrüchte und Meerfische sind entweder über einen IgE-vermittelten Mechanismus gegen Allergene wie Fischproteine oder aber z.B. gegen das Tropomyosin in Krustazeen gerichtet. Nicht selten wird auch die Scombroidintoxikation, eine durch biogene Amine hervorgerufene Histaminintoxikation vor allem auf Thunfisch, beobachtet [37].

Die zugeführten Jodmengen sind in den meisten Nahrungsmittel viel zu gering, um irgendwelche jodassoziierten Überempfindlichkeitsreaktionen auszulösen. Allerdings wurden als Rarität Spättypexantheme auf Nahrungsmittel mit hohem Jodgehalt beschrieben. Das vor allem in Japan konsumierte Seegras wie Kelp und wakame kann zu Jodaufnahme von bis zu $20 \mathrm{mg}$ pro Tag führen [2]. Ein Fall einer sechzigjährigen Frau mit einem pruritischen Erythem nach jodhaltigem Kelp und wakame wurde beschrieben, ein weiterer wird erwähnt [38]. Sie reagierte auch auf ein jodhaltiges Kontrastmittel mit einer Erythrodermie. Prick- und Patchteste waren bis auf einen irritativen Epikutantest auf Povidon-Jod negativ. In der Spätablesung des Pricktestes mit Kaliumjodid trat nach 3 Tagen eine erythematöse Induration auf. Patchteste lösten mit einer Verzögerung von 3 Tagen ein morbiliformes Erythem aus. Lymphozytenstimulationsteste mit Kaliumjodid und Amidotrizoat fielen positiv auf. Somit können ausnahmsweise auch über Nahrungsmittel mit sehr hohen Joddosen erythrodermatische Reaktionen ausgelöst werden, wie es auch für die jodierten Kontrastmittel beschrieben wurde [12,13].

\section{Diskussion}

Die Häufigkeit von unerwünschten Reaktionen auf jodhaltige Präparate ist sehr unterschiedlich. Hochosmolare, ionische Kontrastmittel lösten bei 3-12\%, niederosmolare, nichtionische bei 0,7 bis $3 \%$ milde Sofortreaktionen $[12,19,22,39]$ aus. Schwere Reaktionen traten bei 0,1 bis 0,4 bzw. bei 0,01 bis $0,04 \%$ auf. Spätreaktionen ( $1 \mathrm{~h}$ bis 7 Tage nach Exposition) sind schwieriger zu beziffern, da sie in der Literatur eine heterogene Gruppe darstellen. Kopfschmerzen, Fieber, Schwindel, Nausea, Juckreiz, Urtika- ria und verschiedene Exantheme werden darunter subsummiert, wobei die kutanen Reaktionen am häufigsten sind [12,40,41]. Letztere werden mit ca 0,5 bis $3 \%$ angegeben $[13,40,41]$. Jod spielt hier pathophysiologisch keine gesicherte Rolle (Tab. 2).

Andere Überempfindlichkeitsreaktionen auf Jod selbst scheinen deutlich seltener zu sein. Dies widerspiegelt sich in den Daten, die in einer epidemiologischen Studie nach dem Reaktorunfall von Chernobyl in Polen erhoben worden sind. Orales Kaliumjodid löste bei $1 \%$ der Kinder, und 1,25\% der Erwachsenen leichte Exantheme, Dyspnoe bei 0,1 bzw. 0,6\% aus, während gastrointestinale Symptome mit 3\% bzw 1,5\% etwas häufiger waren [42,43]. Sialoadenitis und Jododerme wurden bei den mehr als 17 Mio. Exponierten nicht beobachtet.

Dies zeigt, dass orale Zufuhr auch von höheren Joddosen relativ gut toleriert wird und dass Überempfindlichkeitsreaktionen auf jodhaltige Produkte sehr selten sind. Wie häufig in der Allergologie, kann nicht von einer "Jodallergie“ an sich gesprochen werden. Die Pathomechanismen sind wie oben beschrieben sehr unterschiedlich und es kann nicht von einer generellen Überempfindlichkeit auf Jod ausgegangen werden (Tab. 2). Patienten mit pseudoallergischen Sofortreaktionen auf Kontrastmittel haben ein erhöhtes Risiko wiederum auf Kontrastmittel zu reagieren. Auch Patienten mit Spättypexanthemen, Jododerm oder Jodsialoadenitis werden auf weitere jodierte Kontrastmittel oder hohe Joddosen rezidivieren. Hingegen sind anaphylaktoide Reaktionen auf jodierte Kontrastmittel oder auf Povidon-Jod nicht auf das Jod selbst zurückzuführen, somit können solche Patienten bei Indikation andere jodhaltige Medikamente erhalten. Auch reichen, allenfalls mit Ausnahme bei Kelp, die mit der Nahrung aufgenommenen Jodmengen nicht aus, um bei Jodsensibilisierten Symptome auszulösen. Eine unerwünschte Reaktion auf ein jodhaltiges Medikament sollte deshalb nach heutigen Standards abgeklärt werden, damit die Betroffenen entsprechend beraten und instruiert werden können, um ihnen nicht wichtige Untersuchungen oder Therapeutika vorzuenthalten oder überflüssige Diäten aufzuerlegen.

\section{Literatur}

${ }^{1}$ Confino-Cohen R, Goldberg A. Safe administration of contrast media: what do physicians know? Ann Allergy Asthma Immunol 2004; 93: $166-170$

${ }^{2}$ Kolb N, Vallorani L, Milanovic N, Stocchi V. Evaluation of marine algae Wakame (Undaria pinnatifida) and Kombu (Laminaria digitata japonica) as food supplements. Food Technol Biotechnol 2004; 42: 57-62

${ }^{3}$ Henzen C, Buess M, Brander L. Die Jod-induzierte Hyperthyreose (Jodbasedow): ein aktuelles Krankheitsbild. Schweiz Med Wochenschr 1999; 129: 658-664

${ }^{4}$ Corazza M, Bulciolu G, Spisani L, Virgili A. Chemical burns following irritant contact with povidone-iodine. Contact Dermatitis 1997; 36: $115-116$

${ }^{5}$ Iijima S, Kuramochi M. Investigation of irritant skin reaction by $10 \%$ povidone-iodine solution after surgery. Dermatology 2002; 204 (Suppl 1): 03-108

${ }^{6}$ Schuetz P, Eriksson U, Christ-Crain M, Zulewski H, Müller B. Amiodaron-induzierte Thyreotoxikose: Eine diagnostische und therapeutische Herausforderung. Praxis 2005; 94: 1397 - 1401

${ }^{7}$ Bloch B. Experimentelle Studien über das Wesen der Jodoformidiosynkrasie. Z Exp Pathologie und Therapie 1911; 9: 509-538

${ }^{8}$ Erdmann S, Hertl M, Merk HF. Allergic contact dermatitis from povidone-iodine. Contact Dermatitis 1999; 40: 331 - 332 
${ }^{9}$ Nishioka K, Seguchi T, Yasuno H, Yamamoto T, Tominaga K. The results of ingredient patch testing in contact dermatitis elicited by povidoneiodine preparations. Contact Dermatitis 2000; 42: 90-94

${ }^{10}$ Lachapelle JM. Allergic contact dermatitis from povidone-iodine: a reevaluation study. Contact Dermatitis 2005; 52: 9-10

${ }^{11}$ Lee SK, Zhai H, Maibach HI. Allergic contact dermatitis from iodine preparations: a conundrum. Contact Dermatitis 2005; 52: 184-187

12 Brockow K, Christiansen C, Kanny G, Clement O, Barbaud A, Bircher A, Dewachter P, Gueant JL, Rodriguez Gueant RM, Mouton-Faivre C, Ring J, Romano A, Sainte-Laudy J, Demoly P, Pichler WJ. Management of hypersensitivity reactions to iodinated contrast media. Allergy 2005; 60: $150-158$

${ }^{13}$ Christiansen C, Pichler WJ, Skotland T. Delayed allergy-like reactions to X-ray contrast media: mechanistic considerations. Eur Radiol 2000; 10: $1965-1975$

${ }^{14}$ Vernassiere C, Trechot P, Commun N, Schmutz JL, Barbaud A. Low negative predictive value of skin tests in investigating delayed reactions to radio-contrast media. Contact Dermatitis 2004; 50: 359-366

15 Ose K, Doue T, Zen K, Hadase M, Sawada T, Azuma A, Matsubara H. „Gadolinium” as an alternative to iodinated contrast media for X-ray angiography in patients with severe allergy. Circ J 2005; 69: 507-509

${ }^{16}$ Remy-Jardin M, Dequiedt P, Ertzbischoff O, Tillie-Leblond I, Bruzzi J, Duhamel A, Remy J. Safety and effectiveness of gadolinium-enhanced multi-detector row spiral CT angiography of the chest: preliminary results in 37 patients with contraindications to iodinated contrast agents. Radiology 2005; 235: 819-826

${ }^{17}$ Häusermann $\mathrm{P}$, Harr T, Bircher AJ. Baboon syndrome resulting from systemic drugs: is there strife between SDRIFE and allergic contact dermatitis syndrome? Contact Dermatitis 2004; 51: 297-310

${ }^{18}$ Bach S, Anner Y, Arnold A, Hohenstein E, Häusermann P, Bircher AJ. Delayed hypersensitivity reactions to iodinated contrast media in 2 patients: unusual manifestation as iodine mumps and flexural exanthem (SDRIFE). Swiss Med Wkly 2005; 135: S14

${ }^{19}$ Dewachter P, Trechot P, Mouton-Faivre C. „Allergie à l'iode“: le point sur la question. Ann Fr Anesth Reanim 2005; 24: 40-52

${ }^{20}$ Adachi A, Fukunaga A, Hayashi K, Kunisada M, Horikawa T. Anaphylaxis to polyvinylpyrrolidone after vaginal application of povidone-iodine. Contact Dermatitis 2003; 48: 133-136

${ }^{21}$ Le Pabic F, Sainte-Laudy J, Blanchard N, Moneret-Vautrin DA. First case of anaphylaxis to iodinated povidone. Allergy 2003; 58: 826-827

${ }^{22}$ Idee JM, Pines E, Prigent P, Corot C. Allergy-like reactions to iodinated contrast agents. A critical analysis. Fundam Clin Pharmacol 2005; 19 : $263-281$

${ }^{23}$ Greenberger PA, Patterson R. The prevention of immediate generalized reactions to radiocontrast media in high-risk patients. J Allergy Clin Immunol 1991; 87: 867-872

${ }^{24}$ Kleeberg L. Toxicodermien II. Jod. In: Jadassohn J (ed). Handbuch der Haut- und Geschlechtskrankheiten IV/2. Berlin: Springer, 1933: 272 297

${ }^{25}$ Plewig G, Strzeminski YA. Jod und Hauterkrankungen. Dtsch Med Wochenschr 1985; 110: $1266-1269$

${ }^{26}$ Miranda-Romero A, Sanchez-Sambucety P, Esquivias Gomez JI, Martinez Fernandez M, Bajo del Pozo C, Aragoneses Fraile H, Garcia-Munoz M. Vegetating iododerma with fatal outcome. Dermatology 1999; 198: $295-297$
${ }^{27}$ Ricci C, Krasovec M, Frenk E. Iodides à l'amiodarone traitées par ciclosporine. Ann Dermatol Venereol 1997; 124: 260 - 263

${ }^{28}$ Vaillant L, Pengloan J, Blanchier D, De Muret A, Lorette G. Iododerma and acute respiratory distress with leucocytoclastic vasculitis following the intravenous injection of contrast medium. Clin Exp Dermatol 1990; 15: $232-233$

${ }^{29}$ Soria C, Allegue F, Espana A, Rocamora A, Harto A, Ledo A. Vegetating iododerma with underlying systemic diseases: report of three cases. J Am Acad Dermatol 1990; 22: 418 - 422

${ }^{30}$ Christensen J. Iodide mumps after intravascular administration of a nonionic contrast medium. Case report and review of the literature. Acta Radiol 1995; 36: $82-84$

${ }^{31}$ Cuéllar J, Hernández F, de Rojas D, Muñoz-Cano A, Giner M, Gómez J, Basomba A. Acute generalised sialoadenitis caused by iodinated radiocontrast media. Report of two cases. Allergol Immunol Clin 2000; 15: 406- 409

${ }^{32}$ Reutter FW, Eugster C. Acute iodine poisoning associated with sialadenitis, allergic vasculitis and conjunctivitis following administration of iodine-containing contrast media. Schweiz Med Wochenschr 1985; 115: $1646-1651$

${ }^{33}$ Curd JG, Milgrom H, Stevenson DD, Mathison DA, Vaughan JH. Potassium iodide sensitivity in four patients with hypocomplementemic vasculitis. Ann Intern Med 1979; 91: 853-857

${ }^{34}$ From E, Thomsen K. Dermatitis herpetiformis. A case provoked by iodine. Br J Dermatol 1974; 91: 221 - 224

${ }^{35}$ Haffenden GP, Blenkinsopp WK, Ring NP, Wojnarowska F, Fry L. The potassium iodide patch test in dermatitis herpetiformis in relation to treatment with a gluten-free diet and dapsone. Br J Dermatol 1980; 103: $313-317$

${ }^{36}$ Burches E, Garcia-Verdegay F, Ferrer M, Pelaez A. Amiodarone-induced angioedema. Allergy 2000; 55: 1199-1200

${ }^{37}$ Bircher A, Schaub N, Scherer K. Der Fang des Tages: Scombrotoxin und Anisakis simplex als Ursache allergieähnlicher Krankheitsbilder. Allergologie 2005; 28: 330-337

${ }^{38}$ Kubota Y, Koga T, Nakayama J. Iodine allergy induced by consumption of iodine-containing food. Contact Dermatitis 2000; 42: 286-287

${ }^{39}$ Katayama H, Yamaguchi K, Kozuka T, Takashima T, Seez P, Matsuura K. Adverse reactions to ionic and nonionic contrast media. A report from the Japanese Committee on the Safety of Contrast Media. Radiology 1990; 175: $621-628$

${ }^{40}$ Hosoya T, Yamaguchi K, Akutsu T, Mitsuhashi Y, Kondo S, Sugai Y, Adachi M. Delayed adverse reactions to iodinated contrast media and their risk factors. Radiat Med 2000; 18: 39-45

${ }^{41}$ Webb JA, Stacul F, Thomsen HS, Morcos SK. Late adverse reactions to intravascular iodinated contrast media. Eur Radiol 2003; 13: 181 -184

42 Nauman J, Wolff J. Iodide prophylaxis in Poland after the Chernobyl reactor accident: benefits and risks. Am J Med 1993; 94: 524-532

${ }^{43}$ Sicherer SH. Risk of severe allergic reactions from the use of potassium iodide for radiation emergencies. J Allergy Clin Immunol 2004; 114: $1395-1397$

${ }^{44}$ Wenlock RW, Buss DH, Moxon RE, Bunton RG. Trace nutrients. 4. Iocline in British food. Br J Nutr 1982; 47: 381 - 390 